P. Kovalenko ${ }^{1, *}$, V. Trokhymets ${ }^{2}$, I. Parnikoza ${ }^{3,4,5}$, Yu. Protsenko ${ }^{2}$, O. Salganskiy ${ }^{6}$, A. Dzhulai ${ }^{3}$, I. Dykyy ${ }^{7}$, M. Nabokin ${ }^{8}$, I. Kozeretska ${ }^{3}$, V. Gorobchyshyn ${ }^{1,2}$

${ }^{1}$ State Institution Institute for Evolutionary Ecology, National Academy of Sciences of Ukraine, Kyiv, 03143, Ukraine

2 Taras Shevchenko National University of Kyiv, Kyiv, 01601, Ukraine

${ }^{3}$ State Institution National Antarctic Scientific Center, Ministry of Education and Science of Ukraine, Kyiv, 01601, Ukraine

${ }^{4}$ Institute of Molecular Biology and Genetics, National Academy of Sciences of Ukraine, Kyiv, 03143, Ukraine

${ }^{5}$ National University of Kyiv-Mohyla Academy, Kyiv, 04070, Ukraine

${ }^{6}$ National University of Life and Environmental Sciences of Ukraine, Kyiv, 03041, Ukraine

${ }^{7}$ Ivan Franko National University of Lviv, Lviv, 79000, Ukraine

${ }^{8}$ Ukrainian Scientific Center of Ecology of the Sea, Ministry of Environment and Natural Resources of Ukraine, Odesa, 65009, Ukraine

* Corresponding author: ilovebiofack@gmail.com

\title{
Current status of Belgica antarctica Jacobs, 1900 (Diptera: Chironomidae) distribution by the data of Ukrainian Antarctic Expeditions
}

\begin{abstract}
Given the recent climate changes and their impact on the Antarctic Peninsula ecosystems, the emergence of invasive species, and increased tourism activity in this region, monitoring changes in the habitats of species native to the Antarctic Peninsula region is necessary. One such species is the Antarctic midge Belgica antarctica (Chironomidae). This insect is endemic to the Antarctic Peninsula and adjacent islands. Thorough studies of changes in the range of this species have not been performed in recent decades. In this study, we present the area of Belgica antarctica according to the collections of biological samples during 2007-2021 (XII, XIV, XVI, XXIV, and XXV Ukrainian Antarctic Expeditions). This Antarctic midge was recorded at 26 localities on the Antarctic Peninsula and 212 localities on 55 surrounding islands between $66^{\circ} 08^{\prime} 38.4^{\prime \prime}$ S, 65 $43^{\prime} 37.1^{\prime \prime}$ W (Cape Even-

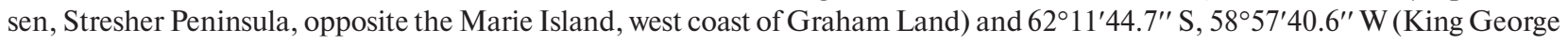
Island, South Shetland Islands) from south-west to north-east.
\end{abstract}

Keywords: Antarctic midge, Antarctic Peninsula, chironomids, endemic species, polar insect

\section{Introduction}

Belgica antarctica Jacobs, 1900 (Diptera: Chironomidae) is chironomid midge, an endemic to the maritime Antarctic (Janetschek, 1970; Sugg et al., 1983; Richard et al., 1994; Convey \& Block, 1996; Allegrucci et al., 2012; Chown \& Convey, 2016; Kozeretska et al., 2021), one of two native species of insects to this region - the other one is Parochlus steinenii Gercke, 1889 (Diptera: Chironomidae), which also occurs in southern South America and sub-Antarctic South Georgia (Convey \& Block, 1996; Allegrucci et al., 2006; Chown 
\& Convey, 2016; Contador et al., 2020; Gañan et al., 2021; Kozeretska et al., 2021).

Belgica antarctica is well-known for its physiological, biochemical, genomic, and life-history features, which help this species to survive in extreme environments (Kozeretska et al., 2021). Traits of the life cycle of this midge are also interesting: unlike most other Chironomidae, it lasts two years (Sugg et al., 1983; Usher \& Edwards, 1984; Convey \& Block, 1996; Rinehart et al., 2006; Kozeretska et al., 2021). The lengthening of the life cycle is a peculiarity of polar invertebrates compared to phylogenetically related species from temperate, tropical, and subtropical regions (Convey, 1996; Søvik, 2002; 2003; Rinehart et al., 2006).

The distribution of $B$. antarctica has been described in few works (Gressitt, 1967; Wirth \& Gressitt, 1967; Peckham, 1971; Convey \& Block, 1996; Allegrucci et al., 2006; Harada et al., 2014). According to the literature, the southernmost confirmed record of this species is Refuge Islands $\left(68^{\circ} 21^{\prime} \mathrm{S}, 67^{\circ} 08^{\prime} \mathrm{W}\right.$; Marguerite Bay) and the northernmost - Elephant Island (61 $\left.{ }^{\circ} 10^{\prime} \mathrm{S}, 55^{\circ} 14^{\prime} \mathrm{W}\right)$ (Usher \& Edwards, 1984). The first published record of $B$. antarctica in the vicinity of Ukrainian Antarctic Akademik Vernadsky station, former British Faraday Station, is dated 1904. The samples were collected from several sites on Booth Island $\left(65^{\circ} 4^{\prime} 48^{\prime \prime} \mathrm{S}, 64^{\circ} 0^{\prime} 0^{\prime \prime} \mathrm{W}\right)$ and Hovgaard Island $\left(65^{\circ} 05^{\prime} \mathrm{S}, 64^{\circ} 05^{\prime} \mathrm{W}\right)$ (Roubard, 1906). It should be noted that the first record of this species on Galindez Island (Argentine Islands, Wilhelm Archipelago, where the Akademik Vernadsky station is located) is dated January 1963 (Wirth \& Gressitt, 1967).

Considering that the ability of a species to adapt to environmental changes is reflected in the area of its distribution (Nunney, 2015), it is possible to expect changes in the distribution of $B$. antarctica. Climate changes have manifested in Antarctic ecosystems (Fowbert \& Smith, 1994; Frenot et al., 2005; Convey \& Smith, 2006; Parnikoza et al., 2009; Turner et al., 2014; Loisel et al., 2017; Sancho et al., 2017; Convey \& Peck, 2019; Bargagli, 2020; Savenets et al., 2020; Bokhorst et al., 2021). Moreover, Kozeretska et al. (2021) proposed to consider B. antarctica as a model organism for studying adaptive mechanisms of survival under climate changes.
The information about the distribution of $B$. antarctica is obsolete, incomplete, and fragmentary, lacking coordinates of most sampling points. Therefore, it is necessary to study the current range of $B$. antarctica to initiate monitoring its changes. Our study is a part of this research.

\section{Materials and methods}

All data represented in this study are based on 323 invertebrate samples made in 2007-2010, 2012, and 2019-2021 (XII, XIV, XVI, XXIV, and XXV Ukrainian Antarctic Expeditions).

Samples were collected by the authors of this paper mostly from locations in the Wilhelm Archipelago and Kyiv Peninsula regions. But some of the materials were collected on several sites in the Palmer Archipelago, South Shetland Islands, Pefaur Peninsula, Arctowski Peninsula, and Graham Land.

Both the eclector (Golub et al., 1980; Protsenko, 2012) and individual catching were used for collecting samples. After it, the samples were preserved in $96 \%$ or $70 \%$ ethanol or $3: 1$ ethanol-acetic acid or ethanol-glycerin fixatives, deep-freezed $\left(-20^{\circ} \mathrm{C}\right)$, and then transported to Ukraine for analysis.

The species was identified according to Wirth \& Gressitt (1967) and Usher \& Edwards (1984), using stereoscopic microscopes: Bresser Advance ICD 10x160x (Germany) and Olympus SZX12 (China).

A distribution map of $B$. antarctica was created using QIGS 3.10.10 (https://www.qgis.org/en/site/forusers/ download.html).

The collected material was also used to perform genetic and cytogenetic (Kovalenko et al., 2021; Michailova et al., 2021a), morphological (Michailova et al., 2021b), biotic and cenotic studies (Polishuk et al., 2009; Dykyy et al., 2012; Caruso et al., 2013; Trokhymets et al., 2014).

\section{Results}

B. antarctica was found in Bryophyte carpet and mat subformation on Sanionia sp. Loeske, 1907 (Amblystegiaceae), Warnstorfia fontinaliopsis (Müll.Hal.) Ochyra, 2001 (Amblystegiaceae), and other short cushion 


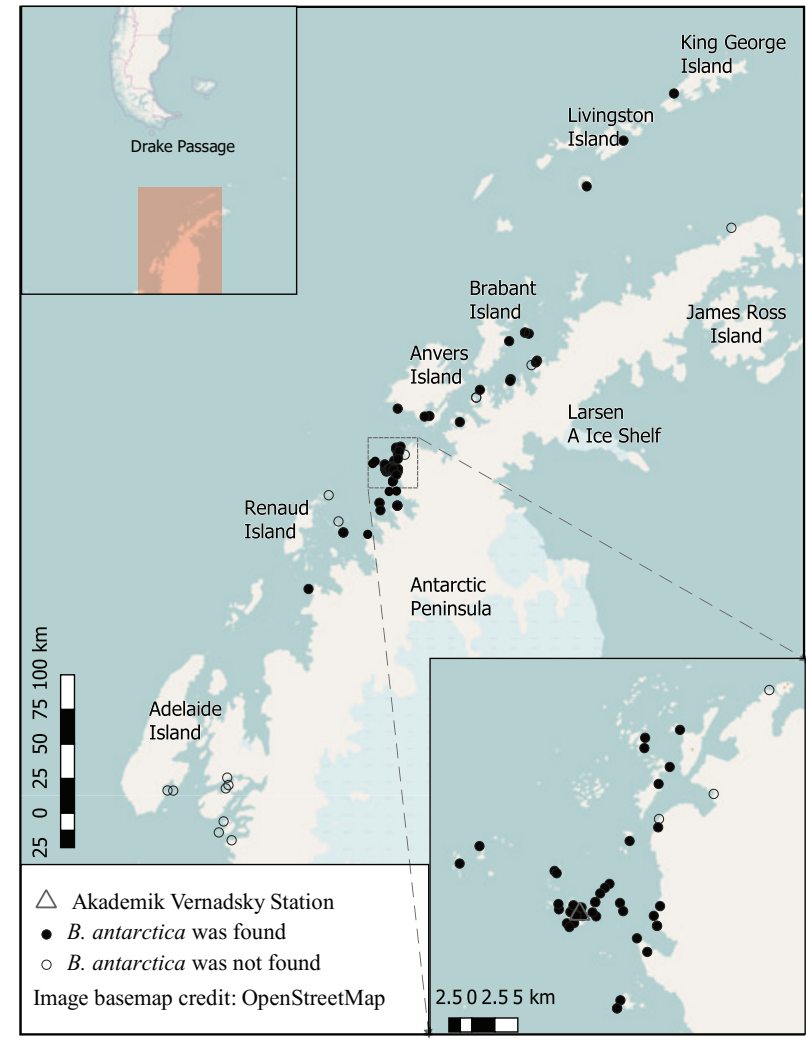

Figure. The distribution of Belgica antarctica in line with the data of Ukrainian Antarctic Expeditions

mosses (in some cases, with add of flowering plants: Deschampsia antarctica È. Desv. 1854 (Poaceae)); alga subformations (Prasiola crispa (Lightfoot) Kützing 1843, (Prasiolaceae); tall moss turf subformations (Polytrichum strictum Menzies ex Brid., 1801 (Polytrichaceae)); short moss turf and cushion subformations (undetermined Bryophytes); nesting and feeding areas of birds: kelp gulls (Larus dominicanus Lichtenstein, 1823 (Laridae)) and cormorants (Phalacrocorax atriceps King, 1828 (Phalacrocoracidae)) (Appendix).

It should be noted that at all sites with $B$. antarctica there were found larvae (mostly - fourth-instar), nevertheless at some sites pupae and adults were also collected.

Based on the results of five Ukrainian Antarctic Expeditions, B. antarctica was found in 238 samples from 212 localities on 55 islands and 26 localities on the Antarctic Peninsula (Figure, Appendix). During these expeditions, approximately 5000 individuals of $B$. antarctica were collected.

According to these data, this midge was recorded

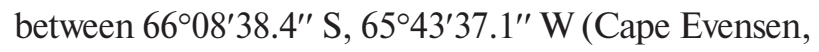
Stresher Peninsula, opposite the Marie Island, west coast of Graham Land) and 62 $11^{\prime} 44.7^{\prime \prime}$ S, 58 $57^{\prime} 40.6^{\prime \prime} \mathrm{W}$ (King George Island, South Shetland Islands) from south-west to north-east.

\section{Discussion}

Many previous works, which included the collections of $B$. antarctica, were indicated large islands without a list of accurate coordinates of the samples collections or archipelagos only, instead of individual islands. That is why it is difficult to say about possible changes in the range of this species. The discrepancies of our data with previous works can be caused by the limitations of our materials, the inability to get the names of all islands from these works, and by lack of precise coordinates in the body of literature. It should be noted that on all islands, individuals of $B$. antarctica were collected where this species had been previously registered. However, we have noted 42 mostly small islands (Wilhelm Archipelago, Palmer Archipelago, South Shetland Islands, and other islands of West coast of Graham Land) where there is no literature data about the presence of $B$. antarctica in their area (see Appendix).

According to the results of the five expeditions, there were no significant changes in the distribution of $B$. antarctica in the more detailed regularly studied regions (Wilhelm Archipelago and Kyiv Peninsula) over the past 14 years. This may be because these studies' main purpose was registering $B$. antarctica in the studied areas rather than monitoring its spread. In recent years there have been changes in the distribution of at least one species-consort of B. antarctica D. antarctica (Fowbert \& Smith, 1994; Parnikoza et al., 2011; Torres-Mellado et al., 2011; Vera et al., 2013). We can assume that they will be reflected in the distribution of this midge.

It is also important to note the appearance of alien species in the Antarctic Peninsula and adjacent islands (Frenot et al., 2005; Hughes et al., 2020; Bokhorst et 
al., 2021); in addition, a surge in tourism activity has been observed in this region in the XXI century (Chwedorzewska et al., 2020; Yevchun et al., 2021). That can affect both ecosystems and separate species' ranges (Chwedorzewska et al., 2020). Due to these facts, it is necessary to keep monitoring the Antarctic biota to identify changes and make forecasts.

Data availability. All data can be found within the article and Appendix.

Author contributions. PK, VG - conceptualization; VT, IP, OS, AD, ID, MN - collecting samples; PK, VT - identification of samples; YuP - map preparation; PK, IP - Appendix preparation; PK, VG writing the first draft of the manuscript, IK - project administration. Each author contributed to the analysis and discussion of the manuscript.

Acknowledgments. We kindly thank two anonymous reviewers for their helpful and constructive comments.

Funding. The current study was performed in the framework of the Ukrainian State Special-Purpose Research Program in Antarctica for 2011-2023.

Conflict of Interest. The authors vouch that they have no conflict of interest.

\section{References}

Allegrucci, G., Carchini, G., Todisco, V., Convey, P., \& Sbordoni, V. (2006). A molecular phylogeny of antarctic chironomidae and its implications for biogeographical history. $\mathrm{Po}$ lar Biology, 29(4), 320-326. http://dx.doi.org/10.1007/s00300005-0056-7

Allegrucci, G., Carchini, G., Convey, P., \& Sbordoni, V. (2012). Evolutionary geographic relationships among orthocladine chironomid midges from maritime Antarctic and subAntarctic islands. Biological Journal of the Linnean Society, 106(2), 258-274. https://doi.org/10.1111/j.1095-8312.2012.01864.x

Bargagli, R. (2020). Terrestrial ecosystems of the Antarctic Peninsula and their responses to climate change and anthropogenic impacts. Ukrainian Antarctic Journal, 2, 84-97. https://doi.org/10.33275/1727-7485.2.2020.656

Bokhorst, S., Convey, P., Casanova-Katny, A., \& Aerts, R. (2021). Warming impacts potential germination of non-native plants on the Antarctic Peninsula. Communications Biology, 4(1), 403. https://doi.org/10.1038/s42003-021-01951-3

Caruso, T., Trokhymets, V., Bargagli, R., \& Convey, P. (2013). Biotic interactions as a structuring force in soil communities: evidence from the micro-arthropods of an Antarctic moss model system. Oecologia, 172(2), 495-503. https://doi.org/10. 1007/s00442-012-2503-9

Chown, S. L., \& Convey, P. (2016). Antarctic Entomology. Annual Review of Entomology, 61(1), 119-137. https://doi.org/ 10.1146/annurev-ento-010715-023537

Chwedorzewska, K. J., Korczak-Abshire, M., \& Znój, A. (2020). Is Antarctica under threat of alien species invasion? Global Change Biology, 26(4), 1942-1943. https://doi.org/10. $1111 / \mathrm{gcb} .15013$

Contador, T., Gañan, M., Bizama, G., Fuentes-Jaque, G., Morales, L., Rendoll, J., Simoes, F., Kennedy, J., Rozzi, R., \& Convey, P. (2020). Assessing distribution shifts and ecophysiological characteristics of the only Antarctic winged midge under climate change scenarios. Scientific Reports, 10(1), 9087. https://doi.org/10.1038/s41598-020-65571-3

Convey, P. (1996). The influence of environmental characteristics on life history attributes of Antarctic terrestrial biota. Biological Reviews, 71(2), 191-225. https://doi.org/10.1111/ j.1469-185X.1996.tb00747.X

Convey, P., \& Block, W. (1996). Antarctic Diptera: Ecology, physiology and distribution. European Journal of Entomology, 93(1), 1-13. https://www.eje.cz/magno/eje/1996/mn1.php

Convey, P., \& Smith, R. I. L. (2006). Responses of terrestrial Antarctic ecosystems to climate change. Plant Ecology, 182(1), 1-10. https://doi.org/10.1007/s11258-005-9022-2

Convey, P., \& Peck, L. S. (2019). Antarctic environmental change and biological responses. Science Advances, 5(11), eaaz0888. https://doi.org/10.1126/sciadv.aaz0888

Download QGIS for your platform. Retrieved November 1, 2021, from https://www.qgis.org/en/site/forusers/download.html

Dykyy, I., Tsaryk, Y., Shydlovskyy, I., Trokhymets, V., \& Holovachov, O. (2012). Cenotic connection land biota Islands Western Antarctic. Ukrainian Antarctic Journal, 10-11, 239256. https://doi.org/10.33275/1727-7485.10-11.2012.304 (In Ukrainian)

Fowbert, J. A., \& Smith, R. I. L. (1994). Rapid population increases in native vascular plants in the Argentine Islands, Antarctic Peninsula. Arctic and Alpine Research, 26(3), 290296. https://doi.org/10.2307/1551941

Frenot, Y., Chown, S. L., Whinam, J., Selkirk, P. M., Convey, P., Skotnicki, M., \& Bergstrom, D. M. (2005). Biological invasions in the Antarctic: extent, impacts and implications. Biological Reviews, 80(1), 45-72. https://doi.org/10.1017/ S1464793104006542

Gañan, M., Contador, T., Rendoll, J., Simoes, F., Pérez, C., Graham, G., Castillo, S., Kennedy, J., \& Convey, P. (2021). Records of Parochlus steinenii in the Maritime Antarctic and sub-Antarctic regions. ZooKeys, 1011, 63-71. https://doi.org/ 10.3897/zookeys.1011.56833

Golub, V. B., Kolesova, D. A., Shurovenkov, Yu. B., \& Elchibaev, A. A. (1980). Entomologicheskie i fitopatologicheskie kollekcii, ih sostavlenie $i$ khranenie [Entomological and phyto- 
pathological collections, their composition and storage]. Izd-vo Voronezh. gos. un-ta. (In Russian)

Gressitt, J. L. (1967). Notes on arthropod populations in the Antarctic Peninsula-South Shetland Islands-South Orkney Islands Area. Antarctic Research Series, 10, 373-391. https:// doi.org/10.1029/AR010p0373

Harada, E., Lee Jr., R. E., Denlinger, D. L., \& Goto, S. G. (2014). Life history traits of adults and embryos of the Antarctic midge Belgica antarctica. Polar Biology, 37(8), 1213-1217. https://doi.org/10.1007/s00300-014-1511-0

Hughes, K. A., Pescott, O. L., Peyton, J., Adriaens, T., Cottier-Cook, E. J., Key, G., Rabitsch, W., Tricarico, E., Barnes, D. K. A., Baxter, N., Belchier, M., Blake, D., Convey, P., Dawson, W., Frohlich, D., Gardiner, L. M., González-Moreno, P., James, R., Malumphy, C., ... \& Roy, H. E. (2020). Invasive non-native species likely to threaten biodiversity and ecosystems in the Antarctic Peninsula region. Global Change Biology, 26(4), 2702-2716. https://doi.org/10.1111/gcb.14938

Janetschek, H. (1970). Environments and ecology of terrestrial arthropods in the high Antarctic. In M. W. Holdgate (Ed.), Antarctic Ecology (vol. 2, pp. 871-885). Academic Press.

Kovalenko, P. A., Serga, S. V., Salganskiy, O. O., \& Kozeretska, I. A. (2021). Analysis of mtDNA haplotypes in Belgica antarctica Jacobs (Diptera, Chironomidae) populations. $X$ International Antarctic Conference Dedicated to the 25th Anniversary of Raising of the National Flag of Ukraine at the Ukrainian Antarctic Akademik Vernadsky Station - X International Antarctic Conference (May 11-13, 2021, Kyiv, Ukraine), p. 46. Kyiv. Retrieved October 20, 2021, from http://uac.gov.ua/wpcontent/uploads/2021/05/Abstracts-X-IAC-2021.pdf

Kozeretska, I., Serga, S., Kovalenko, P., Gorobchyshyn, V., \& Convey, P. (2021). Belgica antarctica (Diptera: Chironomidae): A natural model organism for extreme environments. Insect Science. https://doi.org/10.1111/1744-7917.12925

Loisel, J., Yu, Z., Beilman, D. W., Kaiser, K., \& Parnikoza, I. (2017). Peatland Ecosystem Processes in the Maritime Antarctic During Warm Climates. Scientific Reports, 7(1), 12344. https://doi.org/10.1038/s41598-017-12479-0

Michailova, P., Ilkova, J., Kovalenko, P., Dzhulai, A., \& Kozeretska, I. (2021a). Long-term retainment of some chromosomal inversions in a local population of Belgica antarctica Jacobs (Diptera, Chironomidae). Czech Polar Reports, 11(1), 16-24. https://doi.org/10.5817/CPR2021-1-3

Michailova, P., Ilkova, J., Kovalenko, P. A., Gorobchyshyn, V. A., Kozeretska, I. A. \& Convey, P. (2021b). External morphology of larvae of Belgica antarctica Jacobs, 1900 (Diptera, Chironomidae) obtained from two locations in Maritime Antarctica. Insects, 12(9), 792. https://doi.org/10.3390/ insects 12090792

Nunney, L. (2015). Adapting to a changing environment: modeling the interaction of directional selection and plasticity. Journal of Heredity, 107(1), 15-24. https://doi.org/10.1093/ jhered/esv084
Parnikoza, I., Convey, P., Dykyy, I., Trokhymets, V., Milinevsky, G., Tyschenko, O., Inozemtseva, D., \& Kozeretska, I. (2009). Current status of the Antarctic herb tundra formation in the Central Argentine Islands. Global Change Biology, 15(7), 1685-1693.https://doi.org/10.1111/j.1365-2486.2009.01906.x

Parnikoza, I., Kozeretska, I., \& Kunakh, V. (2011). Vascular Plants of the Maritime Antarctic: Origin and Adaptation. American Journal of Plant Sciences, 2(3), 381-395. https://doi. org/10.4236/ajps.2011.23044

Peckham, V. (1971). Notes on the chironomid midge Belgica antarctica Jacobs at Anvers Island in the Maritime Antarctic. Pacific Insects Monograph, 25, 145-166.

Polishuk, V., Kostikov, I., Taran, N., Voitsitsky, V., Budzanivska, I. G., Khyzhnyak, S., \& Trokhymets, V. (2009). The complex studying of Antarctic biota. Ukrainian Antarctic Journal, 8, 293-301. https://doi.org/10.33275/1727-7485.8.2009.456

Protsenko, Yu. V. (2012). Methodical recommendations for practice "Animal ecology": methods of collecting, processing and preservation of arthropods. Geoprint. (In Ukrainian)

Richard, K. J., Convey, P., \& Block, W. (1994). The terrestrial arthropod fauna of the Byers Peninsula, Livingston Island, South Shetland Islands. Polar Biology, 14(6), 371-379. https://doi.org/10.1007/BF00240257

Rinehart, J. P., Hayward, S. A. L., Elnitsky, M. A., Sandro, L. H., Lee Jr, R. E., \& Denlinger, D. L. (2006). Continuous up-regulation of heat shock proteins in larvae, but not adults, of a polar insect. Proceedings of the National Academy of Sciences of the United States of America, 103(38), 14223-14227. https://doi.org/10.1073/pnas.0606840103

Roubard, M. E. (1906). Diptères. In J. Charcot (Ed.), Expédition Antarctique Francaise (1903-1905) (pp. 11-12). Masson. (In French). Retrieved November 1, 2021, from https://www.biodiversitylibrary.org/page/952344

Sancho, L. G., Pintado, A., Navarro, F., Ramos, M., De Pablo, M. A., Blanquer, J. M., Raggio, J., Valladares, F., \& Green, T. G. A. (2017). Recent Warming and Cooling in the Antarctic Peninsula Region has Rapid and Large Effects on Lichen Vegetation. Scientific Reports, 7(1), 5689. https://doi.org/ 10.1038/s41598-017-05989-4

Savenets, M., Pysarenko, L., \& Pishniak, D. (2020). Microclimatic variations of land surface temperature on Galindez Island (western part of the Antarctic Peninsula). Ukrainian Antarctic Journal, 2, 3-15. https://doi.org/10.33275/17277485.2.2020.648

Søvik, G. (2002). The biology and life history of arctic populations of the littoral mite Ameronothrus lineatus (Acari, Oribatida). Experimental and Applied Acarology, 34(1-2), 3-20. https://doi.org/10.1023/B:APPA.0000044436.80588.96

Søvik, G. (2003). Observations on ovoviviparity and mixedparity mode in Arctic populations of Ameronothrus lineatus (Acari, Oribatida). Acarologia, 43(4), 393-398.

Sugg, P., Edwards, J. S., \& Baust, J. (1983). Phenology and life history of Belgica antarctica, an Antarctic midge (Diptera: 
Chironomidae). Ecological Entomology, 8(1), 105-113. https:// doi.org/10.1111/j.1365-2311.1983.tb00487.x

Torres-Mellado, G. A., Jaña, R., \& Casanova-Katny, M. A. (2011). Antarctic hairgrass expansion in the South Shetland Archipelago and Antarctic Peninsula revisited. Polar Biology, 34(11), 1679. https://doi.org/10.1007/s00300-011-1099-6

Trokhymets, V. N., Iakovenko, N. S., Kovalenko, O. S., \& Dykyy, I. V. (2014). Invertebrate fauna of bryophyte communities of the Petermann Island and the adjacent region of the Antarctic Penninsula. Ukrainian Antarctic Journal, 13, 214-224. https://doi.org/10.33275/1727-7485.13.2014.229 (In Ukrainian)

Turner, J., Barrand, N. E., Bracegirdle, T. J., Convey, P., Hodgson, D. A., Jarvis, M., Jenkins, A., Marshall, G., Meredith, M. P., Roscoe, H., Shanklin, J., French, J., Goosse, H., Guglielmin, M., Gutt, J., Jacobs, S., Kennicutt II, M. C., Masson-Delmotte, V., Mayewski, P., ... \& Klepikov, A. (2014). Antarctic climate change and the environment: An update. Polar Record, 50(3), 237-259. https://doi.org/10.1017/S003 2247413000296
Usher, M. B., \& Edwards, M. (1984). A dipteran from south of the Antarctic Circle: Belgica antarctica (Chironomidae), with a description of its larvae. Biological Journal of the Linnean Society, 23(1), 19-31. https://doi.org/10.1111/j.1095-8312.1984.tb00803.x

Vera, M. L., Fernández-Teruel, T., \& Quesada, A. (2013). Distribution and reproductive capacity of Deschampsia antarctica and Colobanthus quitensis on Byers Peninsula, Livingston Island, South Shetland Islands, Antarctica. Antarctic Science, 25(2), 292-302. https://doi.org/10.1017/S0954102012000995

Wirth, W. W., \& Gressitt, J. L. (1967). Diptera: Chironomidae (Midges). Antarctic Research Series, 10, 197-203. https://doi.org/10.1029/AR010p0197

Yevchun, H., Dykyi, E., Kozeretska, I., Fedchuk, A., Karamushka, V., \& Parnikoza, I. (2021). Minimizing tourist impact on the Argentine Islands ecosystem, Antarctic Peninsula, using visitor site guidelines approach. Ukrainian Antarctic Journal, 1, 98-116. https://doi.org/10.33275/1727-7485.1.2021.669

Received: 22 October 2021 Accepted: 16 December 2021

\author{
П. Коваленко, *, В. Трохимець², І. Парнікоза ${ }^{3,4,5}$, Ю. Проценко², \\ О. Салганський ${ }^{6}$, А. Джулай ${ }^{3}$, I. Дикий ${ }^{7}$, М. Набокін ${ }^{8}$, I. Козерецька ${ }^{3}$, В. Горобчишин ${ }^{1,2}$ \\ ${ }^{1}$ Державна установа Інститут еволюційної екології НАН України, Київ, 03143, Україна \\ ${ }^{2}$ Київський національний університет імені Тараса Шевченка, Київ, 01601, Україна \\ ${ }^{3}$ Державна установа Національний антарктичний науковий центр, \\ Міністерство освіти і науки України, Київ, 01601, Україна \\ ${ }^{4}$ Інститут молекулярної біології і генетики НАН України, Київ, 03143, Україна \\ ${ }^{5}$ Національний університет «Києво-Могилянська академія», Київ, 04070, Україна \\ ${ }^{6}$ Національний університет біоресурсів і природокористування України, \\ Київ, 03041, Україна \\ 7 Львівський національний університет імені Івана Франка, Львів, 79000, Україна \\ ${ }^{8}$ Український науковий центр екології моря, Міністерство захисту довкілля \\ та природних ресурсів України, Одеса, 65009, Україна
}

* Автор для кореспонденції: ilovebiofack@gmail.com

Сучасне поширення Belgica antarctica Jacobs, 1900 (Diptera: Chironomidae) за даними Українських антарктичних експедицій

Реферат. Враховуючи останні зміни клімату та їхній вплив на екосистеми Антарктичного півострова, а також появу інвазивних видів та посилення туристичної активності у цьому регіоні, необхідно відслідковувати зміни у місцях проживання аборигенних для регіону Антарктичного півострова видів. Одним із таких видів є Belgica antarctica з родини дзвінцевих комарів (Chironomidae). Ця комаха є ендеміком Антарктичного півострова та прилеглих островів. Грунтовні дослідження щодо змін поширення зазначеного виду протягом останніх десятиліть не здійснювалися. У цьому дослідженні ми представляємо ареал Belgica antarctica згідно зі зборами біологічних зразків протягом 2007-2021 років (XII, XIV, XVI, XXIV та XXV Українські антарктичні експедиції). Цей антарктичний комар-дзвінець був зафіксований

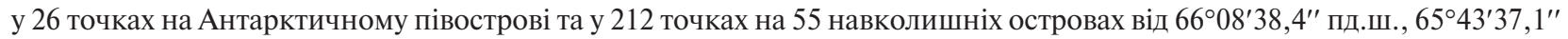
зх.д. (мис Евенсен, півострів Стрешер, навпроти острова Марі, західне узбережжя Землі Греяма) до 62॰11'44,7" пд.ш., 5857'40,6" зх.Д. (острів Кінг-Джордж, Південні Шетландські острови) з південного заходу на північний схід.

Ключові слова: антарктичний комар-дзвінець, Антарктичний півострів, полярна комаха, ендемічний вид, хірономіди 


\section{APPENDIX}

Table 1. Information about sites for which Belgica antarctica was recorded during XII (2007-2008), XIV (2009-2010), XVI (2011-2012), XXIV (2019-2020), and XXV (2020-2021) Ukrainian Antarctic Expeditions. The names of islands on which $B$. antarctica was recorded for the first time (based on the analysis of literature data) by samples collections during the Ukrainian Antarctic Expeditions, highlighted in bold

\begin{tabular}{|c|c|c|c|c|}
\hline Site & Year & Latitude & Longitude & Biotope \\
\hline Galindez Island & 2007 & -65.247726 & -64.240164 & Bryophyte carpet and mat subformation (Sanionia sp.) \\
\hline Galindez Island & 2007 & -65.246200 & -64.249050 & Bryophyte carpet and mat subformation (Sanionia sp.) \\
\hline Galindez Island & 2007 & -65.245650 & -64.252250 & Bryophyte carpet and mat subformation (Sanionia sp.) \\
\hline Galindez Island & 2007 & -65.251133 & -64.242617 & $\begin{array}{l}\text { Bryophyte carpet and mat subformation (Sanionia sp. } \\
\text { and Warnstorfia fontinaliopsis) }\end{array}$ \\
\hline Galindez Island & 2007 & -65.246633 & -64.248833 & $\begin{array}{l}\text { Bryophyte carpet and mat subformation (Warnstorfia } \\
\text { fontinaliopsis, Sanionia sp.) with add of Polytrichum } \\
\text { strictum }\end{array}$ \\
\hline Galindez Island & 2007 & -65.245067 & -64.254717 & $\begin{array}{l}\text { Bryophyte carpet and mat subformation (Sanionia sp.) } \\
\text { with add of Polytrichastrum alpinum and filamentous } \\
\text { algae }\end{array}$ \\
\hline Galindez Island & 2008 & -65.245017 & -64.255500 & $\begin{array}{l}\text { Bryophyte carpet and mat subformation (Warnstorfia } \\
\text { fontinaliopsis, Sanionia sp.) with add of Polytrichum } \\
\text { strictum }\end{array}$ \\
\hline Petermann Island & 2007 & -65.172333 & -64.137833 & Bryophyte carpet and mat subformation (Sanionia sp.) \\
\hline Petermann Island & 2007 & -65.173983 & -64.136367 & Bryophyte carpet and mat subformation (Sanionia sp.) \\
\hline Petermann Island & 2007 & -65.173733 & -64.134900 & Bryophyte carpet and mat subformation (Sanionia sp.) \\
\hline Petermann Island & 2007 & -65.173800 & -64.134667 & Bryophyte carpet and mat subformation (Sanionia sp.) \\
\hline Petermann Island & 2007 & -65.173150 & -64.132350 & Bryophyte carpet and mat subformation (Sanionia sp.) \\
\hline Petermann Island & 2007 & -65.172567 & -64.134700 & Bryophyte carpet and mat subformation (Sanionia sp.) \\
\hline Petermann Island & 2007 & -65.172250 & -64.134900 & Bryophyte carpet and mat subformation (Sanionia sp.) \\
\hline Petermann Island & 2007 & -65.172200 & -64.138633 & Bryophyte carpet and mat subformation (Sanionia sp.) \\
\hline Petermann Island & 2007 & -65.177017 & -64.138033 & Bryophyte carpet and mat subformation (Sanionia sp.) \\
\hline Petermann Island & 2007 & -65.176017 & -64.140000 & Bryophyte carpet and mat subformation (Sanionia sp.) \\
\hline Petermann Island & 2007 & -65.175733 & -64.140750 & Bryophyte carpet and mat subformation (Sanionia sp.) \\
\hline Petermann Island & 2007 & -65.178217 & -64.144117 & Bryophyte carpet and mat subformation (Sanionia sp.) \\
\hline Petermann Island & 2007 & -65.178200 & -64.143483 & Bryophyte carpet and mat subformation (Sanionia sp.) \\
\hline Petermann Island & 2007 & -65.177167 & -64.145183 & Bryophyte carpet and mat subformation (Sanionia sp.) \\
\hline Petermann Island & 2007 & -65.177100 & -64.143633 & Bryophyte carpet and mat subformation (Sanionia sp.) \\
\hline Petermann Island & 2007 & -65.174850 & -64.141250 & Bryophyte carpet and mat subformation (Sanionia sp.) \\
\hline Petermann Island & 2008 & -65.165767 & -64.152033 & Bryophyte carpet and mat subformation (Sanionia sp.) \\
\hline Petermann Island & 2008 & -65.172533 & -64.139050 & Bryophyte carpet and mat subformation (Sanionia sp.) \\
\hline Rasmussen Point & 2007 & -65.247483 & -64.084683 & Bryophyte carpet and mat subformation (Sanionia sp.) \\
\hline Fanfare Island & 2007 & -65.216500 & -64.188267 & Bryophyte carpet and mat subformation (Sanionia sp.) \\
\hline Fanfare Island & 2007 & -65.215967 & -64.189567 & Bryophyte carpet and mat subformation (Sanionia sp.) \\
\hline
\end{tabular}


P. Kovalenko, V. Trokhymets, I. Parnikoza et al.: Distribution of B. antarctica by the data of UAES

Continuation of Table 1

\begin{tabular}{|c|c|c|c|c|}
\hline Site & Year & Latitude & Longitude & Biotope \\
\hline Irizar Island & 2007 & -65.220467 & -64.196633 & Bryophyte carpet and mat subformation (Sanionia sp.) \\
\hline Irizar Island & 2007 & -65.220383 & -64.196783 & Bryophyte carpet and mat subformation (Sanionia sp.) \\
\hline Irizar Island & 2007 & -65.220317 & -64.198833 & Bryophyte carpet and mat subformation (Sanionia sp.) \\
\hline Uruguay Island & 2007 & -65.247967 & -64.219450 & Bryophyte carpet and mat subformation (Sanionia sp.) \\
\hline Berthelot Island & 2007 & -65.329217 & -64.162767 & Bryophyte carpet and mat subformation (Sanionia sp.) \\
\hline Black Island & 2007 & -65.257867 & -64.283583 & Bryophyte carpet and mat subformation (Sanionia sp.) \\
\hline Black Island & 2007 & -65.257683 & -64.278683 & Bryophyte carpet and mat subformation (Sanionia sp.) \\
\hline Leopard Island & 2007 & -65.254783 & -64.287783 & Bryophyte carpet and mat subformation (Sanionia sp.) \\
\hline Leopard Island & 2007 & -65.254867 & -64.287000 & Bryophyte carpet and mat subformation (Sanionia sp.) \\
\hline Booth Island & 2007 & -65.063700 & -64.043050 & Bryophyte carpet and mat subformation (Sanionia sp.) \\
\hline Booth Island & 2007 & -65.064233 & -64.040033 & Bryophyte carpet and mat subformation (Sanionia sp.) \\
\hline Skua Island & 2007 & -65.254383 & -64.271033 & Bryophyte carpet and mat subformation (Sanionia sp.) \\
\hline Skua Island & 2007 & -65.252333 & -64.254250 & Bryophyte carpet and mat subformation (Sanionia sp.) \\
\hline Hovgaard Island & 2007 & -65.235250 & -64.158383 & Bryophyte carpet and mat subformation (Sanionia sp.) \\
\hline Hovgaard Island & 2007 & -65.234533 & -64.163300 & Bryophyte carpet and mat subformation (Sanionia sp.) \\
\hline Hovgaard Island & 2007 & -65.235033 & -64.163733 & Bryophyte carpet and mat subformation (Sanionia sp.) \\
\hline Hovgaard Island & 2007 & -65.233700 & -64.161967 & Bryophyte carpet and mat subformation (Sanionia sp.) \\
\hline Hovgaard Island & 2007 & -65.234850 & -64.157833 & Bryophyte carpet and mat subformation (Sanionia sp.) \\
\hline Cape Tuxen & 2007 & -65.269250 & -64.124050 & Bryophyte carpet and mat subformation (Sanionia sp.) \\
\hline Pléneau Island & 2007 & -65.099833 & -64.053767 & Bryophyte carpet and mat subformation (Sanionia sp.) \\
\hline Darboux Island & 2008 & -65.410700 & -64.234217 & Bryophyte carpet and mat subformation (Sanionia sp.) \\
\hline Darboux Island & 2008 & -65.410567 & -64.234983 & Bryophyte carpet and mat subformation (Sanionia sp.) \\
\hline Cape Perez & 2008 & -65.404133 & -64.099867 & Bryophyte carpet and mat subformation (Sanionia sp.) \\
\hline $\begin{array}{l}\text { un-named island of the } \\
\text { Lippmann Islands }\end{array}$ & 2008 & -65.498033 & -64.413117 & Bryophyte carpet and mat subformation (Sanionia sp.) \\
\hline $\begin{array}{l}\text { cape close to Mount } \\
\text { Waugh, Graham Land }\end{array}$ & 2008 & -65.518833 & -64.082033 & Bryophyte carpet and mat subformation (Sanionia sp.) \\
\hline $\begin{array}{l}\text { un-named island }(1)^{*} \\
\text { of The Barchans }\end{array}$ & 2008 & -65.241217 & -64.306433 & Bryophyte carpet and mat subformation (Sanionia sp.) \\
\hline $\begin{array}{l}\text { un-named island (2) } \\
\text { of The Barchans }\end{array}$ & 2008 & -65.236083 & -64.307700 & Bryophyte carpet and mat subformation (Sanionia sp.) \\
\hline $\begin{array}{l}\text { un-named island, Cruls } \\
\text { Islands }\end{array}$ & 2008 & -65.196750 & -64.538667 & Bryophyte carpet and mat subformation (Sanionia sp.) \\
\hline $\begin{array}{l}\text { un-named island, Cruls } \\
\text { Islands }\end{array}$ & 2008 & -65.190317 & -64.537333 & Bryophyte carpet and mat subformation (Sanionia sp.) \\
\hline $\begin{array}{l}\text { un-named island, Roca } \\
\text { Islands }\end{array}$ & 2008 & -65.178983 & -64.492017 & Bryophyte carpet and mat subformation (Sanionia sp.) \\
\hline Galindez Island & 2020 & -65.247603 & -64.243728 & Alga subformation (Prasiola crispa) \\
\hline Petermann Island & 2020 & -65.176912 & -64.138783 & $\begin{array}{l}\text { Bryophyte carpet and mat subformation (Sanionia sp.) } \\
\text { with green macroalgae (Prasiola crispa) and penguin } \\
\text { feathers }\end{array}$ \\
\hline
\end{tabular}




\begin{tabular}{|c|c|c|c|c|}
\hline Site & Year & Latitude & Longitude & Biotope \\
\hline Petermann Island & 2020 & -65.178403 & -64.145205 & $\begin{array}{l}\text { Bryophyte carpet and mat subformation (Sanionia sp.) } \\
\text { with green macroalgae (Prasiola crispa) and penguin } \\
\text { feathers }\end{array}$ \\
\hline Petermann Island & 2020 & -65.177362 & -64.144209 & $\begin{array}{l}\text { Bryophyte carpet and mat subformation (Sanionia sp.) } \\
\text { and penguin feathers }\end{array}$ \\
\hline Petermann Island & 2020 & -65.173955 & -64.138582 & $\begin{array}{l}\text { Bryophyte carpet and mat subformation (Sanionia } \text { sp.) } \\
\text { with green macroalgae (Prasiola crispa) and penguin } \\
\text { feathers }\end{array}$ \\
\hline Petermann Island & 2020 & -65.172415 & -64.137016 & Bryophyte carpet and mat subformation (Sanionia sp.) \\
\hline $\begin{array}{l}\text { un-named island (1) } \\
\text { of The Barchans }\end{array}$ & 2020 & -65.241830 & -64.300590 & $\begin{array}{l}\text { Bryophyte carpet and mat subformation (Sanionia sp.) } \\
\text { and green macroalgae (Prasiola crispa) }\end{array}$ \\
\hline $\begin{array}{l}\text { un-named island (1) } \\
\text { of The Barchans }\end{array}$ & 2020 & -65.241830 & -64.300590 & Tall moss turf subformation (Polytrichum strictum) \\
\hline $\begin{array}{l}\text { Central of the Three Little } \\
\text { Pigs Islands }\end{array}$ & 2020 & -65.242510 & -64.273960 & $\begin{array}{l}\text { Bryophyte carpet and mat subformation (Sanionia sp.) } \\
\text { and limpet shells }\end{array}$ \\
\hline $\begin{array}{l}\text { Central of the Three Little } \\
\text { Pigs Islands }\end{array}$ & 2020 & -65.242160 & -64.274410 & $\begin{array}{l}\text { Nesting area of Larus dominicanus: Sanionia sp. and } \\
\text { other short cushion moss, limpet shells }\end{array}$ \\
\hline $\begin{array}{l}\text { Eastern of the Three Little } \\
\text { Pigs Islands }\end{array}$ & 2020 & -65.242420 & -64.271920 & $\begin{array}{l}\text { Bryophyte carpet and mat subformation (Sanionia sp. } \\
\text { and Warnstorfia fontinaliopsis) and limpet shells }\end{array}$ \\
\hline $\begin{array}{l}\text { Western of the Three Little } \\
\text { Pigs Islands }\end{array}$ & 2020 & -65.243763 & -64.279475 & $\begin{array}{l}\text { Bryophyte carpet and mat subformation (Sanionia sp.) } \\
\text { and green macroalgae (Prasiola crispa) }\end{array}$ \\
\hline Skua Island & 2020 & -65.255110 & -64.274800 & $\begin{array}{l}\text { Nesting or feeding area of Larus dominicanus: food } \\
\text { remnants of L. dominicanus }\end{array}$ \\
\hline $\begin{array}{l}\text { un-named island close to } \\
\text { Winter Island }\end{array}$ & 2020 & -65.254420 & -64.274930 & $\begin{array}{l}\text { Bryophyte carpet and mat subformation (Sanionia sp. } \\
\text { and Deschampsia antarctica) }\end{array}$ \\
\hline $\begin{array}{l}\text { un-named island close to } \\
\text { Winter Island }\end{array}$ & 2020 & -65.254420 & -64.274930 & Bryophyte carpet and mat subformation (Sanionia sp.) \\
\hline Winter Island & 2020 & -65.249490 & -64.272590 & $\begin{array}{l}\text { Nesting or feeding area of Larus dominicanus: Warnstor- } \\
\text { fia fontinaliopsis and limpet shells }\end{array}$ \\
\hline Winter Island & 2020 & -65.248667 & -64.270233 & Bryophyte carpet and mat subformation (Sanionia sp.) \\
\hline Indicator Island & 2020 & -65.246020 & -64.264860 & Bryophyte carpet and mat subformation (Sanionia sp.) \\
\hline $\begin{array}{l}\text { un-named biggest } \\
\text { southernmost island } \\
\text { of Forge Islands }\end{array}$ & 2020 & -65.236860 & -64.277180 & Bryophyte carpet and mat subformation (Sanionia sp.) \\
\hline $\begin{array}{l}\text { un-named island (2), Forge } \\
\text { Islands }\end{array}$ & 2020 & -65.237240 & -64.272730 & Bryophyte carpet and mat subformation (Sanionia sp.) \\
\hline $\begin{array}{l}\text { un-named island, Roca } \\
\text { Islands }\end{array}$ & 2020 & -65.178850 & -64.491890 & Bryophyte carpet and mat subformation (Sanionia sp.) \\
\hline $\begin{array}{l}\text { un-named island, Roca } \\
\text { Islands }\end{array}$ & 2020 & -65.178850 & -64.491890 & Alga subformation (Prasiola crispa) \\
\hline $\begin{array}{l}\text { un-named island, Roca } \\
\text { Islands }\end{array}$ & 2020 & -65.179830 & -64.492310 & $\begin{array}{l}\text { Bryophyte carpet and mat subformation } \\
\text { (Warnstorfia fontinaliopsis) and green macroalgae } \\
\text { (Prasiola crispa) }\end{array}$ \\
\hline Galindez Island & 2020 & -65.244850 & -64.255610 & $\begin{array}{l}\text { Bryophyte carpet and mat subformation (Sanionia sp.) } \\
\text { and green macroalgae (Prasiola crispa) }\end{array}$ \\
\hline
\end{tabular}


P. Kovalenko, V. Trokhymets, I. Parnikoza et al.: Distribution of B. antarctica by the data of UAES

Continuation of Table 1

\begin{tabular}{|c|c|c|c|c|}
\hline Site & Year & Latitude & Longitude & Biotope \\
\hline Galindez Island & 2020 & -65.244850 & -64.255610 & Bryophyte carpet and mat subformation (Sanionia sp.) \\
\hline Galindez Island & 2020 & -65.244850 & -64.255610 & Alga subformation (Prasiola crispa) and penguin feathers \\
\hline Galindez Island & 2020 & -65.244850 & -64.255610 & $\begin{array}{l}\text { Bryophyte carpet and mat subformation (Sanionia sp. } \\
\text { and Deschampsia antarctica) }\end{array}$ \\
\hline Galindez Island & 2020 & -65.245060 & -64.252640 & $\begin{array}{l}\text { Nesting or feeding area of Larus dominicanus: Sanionia } \\
\text { sp., other short cushion moss and limpet shells }\end{array}$ \\
\hline Galindez Island & 2020 & -65.245060 & -64.252640 & $\begin{array}{l}\text { Nesting or feeding area of Larus dominicanus: Descham- } \\
\text { psia antarctica }\end{array}$ \\
\hline Galindez Island & 2020 & -65.245060 & -64.252640 & $\begin{array}{l}\text { Nesting or feeding area of Larus dominicanus: bryophy- } \\
\text { te carpet and mat subformation (Sanionia sp.), and lim- } \\
\text { pet shells }\end{array}$ \\
\hline Galindez Island & 2020 & -65.245500 & -64.249520 & Bryophyte carpet and mat subformation (Sanionia sp.) \\
\hline Galindez Island & 2020 & -65.247480 & -64.252800 & $\begin{array}{l}\text { Bryophyte carpet and mat subformation (Sanionia sp.) } \\
\text { with add of Polytrichum strictum }\end{array}$ \\
\hline Galindez Island & 2020 & -65.247480 & -64.252800 & $\begin{array}{l}\text { Nesting area of Larus dominicanus: Warnstorfia fonti- } \\
\text { naliopsis, Polytrichum strictum, and limpet shells }\end{array}$ \\
\hline Galindez Island & 2020 & -65.247480 & -64.252800 & $\begin{array}{l}\text { Bryophyte carpet and mat subformation } \\
\text { (Deschampsia antarctica and Sanionia sp.) }\end{array}$ \\
\hline Galindez Island & 2020 & -65.248380 & -64.245840 & $\begin{array}{l}\text { Bryophyte carpet and mat subformation (Sanionia sp. } \\
\text { and Warnstorfia fontinaliopsis) }\end{array}$ \\
\hline Galindez Island & 2020 & -65.248380 & -64.245840 & $\begin{array}{l}\text { Nesting or feeding area of Larus dominicanus: Des- } \\
\text { champsia antarctica and Sanionia sp. }\end{array}$ \\
\hline Irizar Island & 2020 & -65.219390 & -64.199790 & Bryophyte carpet and mat subformation (Sanionia sp.) \\
\hline Irizar Island & 2020 & -65.219390 & -64.199790 & Nest of Larus dominicanus: Sanionia sp. \\
\hline $\begin{array}{l}\text { un-named island of the } \\
\text { Argentine Islands }\end{array}$ & 2020 & -65.225710 & -64.209980 & Bryophyte carpet and mat subformation (Sanionia sp.) \\
\hline Uruguay Island & 2020 & -65.231770 & -64.223380 & $\begin{array}{l}\text { Nest area of Phalacrocorax atriceps: Sanionia sp. and } \\
\text { other short cushion moss }\end{array}$ \\
\hline Uruguay Island & 2020 & -65.231740 & -64.223530 & $\begin{array}{l}\text { Nest of Phalacrocorax atriceps: Warnstorfia fontinalio- } \\
\text { psis, seaweed, and cormorant feathers }\end{array}$ \\
\hline Uruguay Island & 2020 & -65.232810 & -64.222490 & Bryophyte carpet and mat subformation (Sanionia sp.) \\
\hline $\begin{array}{l}\text { Lamya Island (Dannebrog } \\
\text { Islands) }\end{array}$ & 2020 & -65.084430 & -64.106710 & Bryophyte carpet and mat subformation (Sanionia sp.) \\
\hline $\begin{array}{l}\text { Lamya Island (Dannebrog } \\
\text { Islands) }\end{array}$ & 2020 & -65.084260 & -64.113300 & $\begin{array}{l}\text { Nesting or feeding area of Larus dominicanus: Sanionia } \\
\text { sp., other short cushion moss, and limpet shells }\end{array}$ \\
\hline $\begin{array}{l}\text { Lamya Island (Dannebrog } \\
\text { Islands) }\end{array}$ & 2020 & -65.084150 & -64.113210 & $\begin{array}{l}\text { Bryophyte carpet and mat subformation (Sanionia sp. } \\
\text { and Warnstorfia fontinaliopsis) and penguin feathers }\end{array}$ \\
\hline $\begin{array}{l}\text { Elisabethinsel (Dannebrog } \\
\text { Islands) }\end{array}$ & 2020 & -65.075640 & -64.099350 & $\begin{array}{l}\text { Nesting or feeding area of Larus dominicanus: Sanionia } \\
\text { sp., limpet shells and fur of seals }\end{array}$ \\
\hline $\begin{array}{l}\text { Elisabethinsel (Dannebrog } \\
\text { Islands) }\end{array}$ & 2020 & -65.074360 & -64.104940 & Bryophyte carpet and mat subformation (Sanionia sp.) \\
\hline Booth Island & 2020 & -65.066680 & -64.034180 & $\begin{array}{l}\text { Bryophyte carpet and mat subformation (Sanionia sp.) } \\
\text { and penguin feathers }\end{array}$ \\
\hline Booth Island & 2020 & -65.065970 & -64.034360 & $\begin{array}{l}\text { Bryophyte carpet and mat subformation (Sanionia sp. } \\
\text { and other short cushion moss) }\end{array}$ \\
\hline
\end{tabular}


Continuation of Table 1

\begin{tabular}{|c|c|c|c|c|}
\hline Site & Year & Latitude & Longitude & Biotope \\
\hline Booth Island & 2020 & -65.065970 & -64.034360 & $\begin{array}{l}\text { Bryophyte carpet and mat subformation (Sanionia sp. } \\
\text { and other short cushion moss) }\end{array}$ \\
\hline Booth Island & 2020 & -65.066660 & -64.023450 & $\begin{array}{l}\text { Nest of Phalacrocorax atriceps: limpet shells and } \\
\text { penguin feathers }\end{array}$ \\
\hline Nob Island & 2020 & -65.203920 & -64.316790 & Bryophyte carpet and mat subformation (Sanionia sp.) \\
\hline $\begin{array}{l}\text { un-named island close to } \\
\text { Nob Island }\end{array}$ & 2020 & -65.206160 & -64.311780 & Bryophyte carpet and mat subformation (Sanionia sp.) \\
\hline Galindez Island & 2020 & -65.247990 & -64.242520 & $\begin{array}{l}\text { Bryophyte carpet and mat subformation (Sanionia sp., } \\
\text { Warnstorfia fontinaliopsis, and other short cushion } \\
\text { moss) and limpet shells }\end{array}$ \\
\hline Galindez Island & 2020 & -65.247500 & -64.241250 & $\begin{array}{l}\text { Bryophyte carpet and mat subformation (Deschampsia } \\
\text { antarctica and Sanionia sp.) }\end{array}$ \\
\hline Hovgaard Island & 2020 & -65.119340 & -64.073410 & $\begin{array}{l}\text { Nest of Larus dominicanus: Sanionia sp., Warnstorfia } \\
\text { fontinaliopsis, Polytrichum strictum, Usnea antarctica, } \\
\text { and feather }\end{array}$ \\
\hline Hovgaard Island & 2020 & -65.119340 & -64.073410 & $\begin{array}{l}\text { Short moss turf and cushion (undetermined Bryo- } \\
\text { phytes) }\end{array}$ \\
\hline Hovgaard Island & 2020 & -65.119430 & -64.072650 & Tall moss turf subformation (Polytrichum strictum) \\
\hline Hovgaard Island & 2020 & -65.119430 & -64.072650 & $\begin{array}{l}\text { Bryophyte carpet and mat subformation with } \\
\text { Deschampsia antarctica }\end{array}$ \\
\hline Hovgaard Island & 2020 & -65.120210 & -64.069930 & $\begin{array}{l}\text { Bryophyte carpet and mat subformation } \\
\text { (Warnstorfia fontinaliopsis) }\end{array}$ \\
\hline Hovgaard Island & 2020 & -65.119960 & -64.069160 & $\begin{array}{l}\text { Bryophyte carpet and mat subformation } \\
\text { (Warnstorfia fontinaliopsis) }\end{array}$ \\
\hline Hovgaard Island & 2020 & -65.120250 & -64.066670 & Bryophyte carpet and mat subformation (Sanionia sp.) \\
\hline Hovgaard Island & 2020 & -65.131290 & -64.124110 & $\begin{array}{l}\text { Bryophyte carpet and mat subformation (Sanionia sp.) } \\
\text { and green macroalgae Prasiola crispa }\end{array}$ \\
\hline Hovgaard Island & 2020 & -65.131320 & -64.121450 & Bryophyte carpet and mat subformation (Sanionia sp.) \\
\hline Petermann Island & 2020 & -65.165860 & -64.143990 & Bryophyte carpet and mat subformation (Sanionia sp.) \\
\hline Petermann Island & 2020 & -65.165860 & -64.143990 & $\begin{array}{l}\text { Tall moss turf subformation (Polytrichum strictum and } \\
\text { other short cushion moss) }\end{array}$ \\
\hline Petermann Island & 2020 & -65.165860 & -64.143990 & $\begin{array}{l}\text { Bryophyte carpet and mat subformation } \\
\text { (Deschampsia antarctica and Sanionia } \mathrm{sp} . \text {.) }\end{array}$ \\
\hline Petermann Island & 2020 & -65.165410 & -64.148170 & $\begin{array}{l}\text { Bryophyte carpet and mat subformation (Sanionia sp.) } \\
\text { and green macroalgae (Prasiola crispa) }\end{array}$ \\
\hline Lahille Island & 2020 & -65.533430 & -64.328680 & $\begin{array}{l}\text { Bryophyte carpet and mat subformation (Sanionia sp. } \\
\text { and Warnstorfia fontinaliopsis) }\end{array}$ \\
\hline $\begin{array}{l}\text { Mount Waugh, Graham } \\
\text { Land }\end{array}$ & 2020 & -65.518140 & -64.083470 & $\begin{array}{l}\text { Bryophyte carpet and mat subformation (Warnstorfia } \\
\text { fontinaliopsis) and other short cushion moss }\end{array}$ \\
\hline $\begin{array}{l}\text { Mount Waugh, Graham } \\
\text { Land }\end{array}$ & 2020 & -65.519090 & -64.082280 & $\begin{array}{l}\text { Bryophyte carpet and mat subformation (Sanionia sp., } \\
\text { Warnstorfia fontinaliopsis) with add of } \\
\text { Polytrichum strictum }\end{array}$ \\
\hline Cape Perez & 2020 & -65.404130 & -64.100000 & $\begin{array}{l}\text { Bryophyte carpet and mat subformation } \\
\text { (Warnstorfia fontinaliopsis) }\end{array}$ \\
\hline
\end{tabular}


P. Kovalenko, V. Trokhymets, I. Parnikoza et al.: Distribution of B. antarctica by the data of UAES

Continuation of Table 1

\begin{tabular}{|c|c|c|c|c|}
\hline Site & Year & Latitude & Longitude & Biotope \\
\hline Darboux Island & 2020 & -65.395267 & -64.215683 & $\begin{array}{l}\text { Bryophyte carpet and mat subformation (Sanionia sp., } \\
\text { Warnstorfia fontinaliopsis) and other short cushion } \\
\text { moss, and limpet shells }\end{array}$ \\
\hline Cape Perez & 2020 & -65.407730 & -64.097250 & Nesting or feeding area of Larus dominicanus: Sanionia sp. \\
\hline Leopard Island & 2020 & -65.254860 & -64.286860 & Bryophyte carpet and mat subformation (Sanionia sp.) \\
\hline Leopard Island & 2020 & -65.255060 & -64.286140 & $\begin{array}{l}\text { Bryophyte carpet and mat subformation (Sanionia sp.) } \\
\text { and other short cushion moss, limpet shells, and fish } \\
\text { remains }\end{array}$ \\
\hline Black Island & 2020 & -65.258450 & -64.278180 & $\begin{array}{l}\text { Nesting or feeding area of Larus dominicanus: penguin } \\
\text { feathers, limpet shells, Prasiola crispa, Warnstorfia } \\
\text { fontinaliopsis, and fur of seals }\end{array}$ \\
\hline Black Island & 2020 & -65.257680 & -64.283500 & $\begin{array}{l}\text { Nesting or feeding area of Larus dominicanus: penguin } \\
\text { feathers, limpet shells, Prasiola crispa, Warnstorfia } \\
\text { fontinaliopsis, and fur of seals }\end{array}$ \\
\hline Black Island & 2020 & -65.258340 & -64.282120 & Bryophyte carpet and mat subformation (Sanionia sp.) \\
\hline Black Island & 2020 & -65.257890 & -64.284620 & Bryophyte carpet and mat subformation (Sanionia sp.) \\
\hline $\begin{array}{l}\text { un-named island (3) } \\
\text { of The Barchans }\end{array}$ & 2020 & -65.237010 & -64.311910 & $\begin{array}{l}\text { Short moss turf and cushion (undetermined } \\
\text { Bryophytes), limpet shells, and wool of fur seals }\end{array}$ \\
\hline Galindez Island & 2020 & -65.247650 & -64.250740 & $\begin{array}{l}\text { Tall moss turf subformation (Polytrichum strictum and } \\
\text { other short cushion moss) }\end{array}$ \\
\hline Torgersen Island & 2020 & -64.771820 & -64.074450 & Bryophyte carpet and mat subformation (Sanionia sp.) \\
\hline Torgersen Island & 2020 & -64.771720 & -64.075670 & Alga subformation (Prasiola crispa) \\
\hline Torgersen Island & 2020 & -64.771760 & -64.075530 & Bryophyte carpet and mat subformation (Sanionia sp.) \\
\hline Cape Tuxen & 2020 & -65.267660 & -64.115930 & Bryophyte carpet and mat subformation (Sanionia sp.) \\
\hline Cape Tuxen & 2020 & -65.267660 & -64.115930 & $\begin{array}{l}\text { Tall moss turf subformation (Polytrichum strictum) with } \\
\text { add of Sanionia sp. }\end{array}$ \\
\hline Cape Tuxen & 2020 & -65.267040 & -64.115960 & Bryophyte carpet and mat subformation (Sanionia sp.) \\
\hline Rasmussen Island & 2020 & -65.257190 & -64.077070 & $\begin{array}{l}\text { Nesting or feeding area of Larus dominicanus: Warns- } \\
\text { torfia fontinaliopsis, Sanionia sp., Deschampsia antarc- } \\
\text { tica, limpet shells, fragment of fish, feathers }\end{array}$ \\
\hline Rasmussen Island & 2020 & -65.256990 & -64.078890 & Bryophyte carpet and mat subformation (Sanionia sp.) \\
\hline Pléneau Island & 2020 & -65.102800 & -64.047417 & $\begin{array}{l}\text { Bryophyte carpet and mat subformation (Warnstorfia } \\
\text { fontinaliopsis) with green macroalgae (Prasiola crispa) }\end{array}$ \\
\hline Pléneau Island & 2020 & -65.102800 & -64.047417 & Bryophyte carpet and mat subformation (Sanionia sp.) \\
\hline Pléneau Island & 2020 & -65.102800 & -64.047417 & Bryophyte carpet and mat subformation (Sanionia sp.) \\
\hline $\begin{array}{l}\text { un-named island close to } \\
\text { Berthelot Island }\end{array}$ & 2020 & -65.336960 & -64.170440 & Alga subformation (Prasiola crispa) \\
\hline $\begin{array}{l}\text { un-named island close to } \\
\text { Berthelot Island }\end{array}$ & 2020 & -65.336960 & -64.170440 & Bryophyte carpet and mat subformation (Sanionia sp.) \\
\hline $\begin{array}{l}\text { un-named island close to } \\
\text { Berthelot Island }\end{array}$ & 2020 & -65.336740 & -64.173870 & Bryophyte carpet and mat subformation (Sanionia sp.) \\
\hline Grotto Island & 2020 & -65.239370 & -64.253620 & $\begin{array}{l}\text { Nesting or feeding area of Larus dominicanus: Sanionia } \\
\text { sp., other short cushion moss, green algae (Prasiola } \\
\text { crispa), and limpet shells }\end{array}$ \\
\hline
\end{tabular}




\begin{tabular}{|c|c|c|c|c|}
\hline Site & Year & Latitude & Longitude & Biotope \\
\hline Grotto Island & 2020 & -65.238950 & -64.256820 & $\begin{array}{l}\text { Bryophyte carpet and mat subformation (Sanionia sp. } \\
\text { and Warnstorfia fontinaliopsis) }\end{array}$ \\
\hline Rasmussen Point & 2020 & -65.247810 & -64.084690 & $\begin{array}{l}\text { Bryophyte carpet and mat subformation (Sanionia sp.) } \\
\text { with green macroalgae (Prasiola crispa) }\end{array}$ \\
\hline Winter Island & 2020 & -65.247500 & -64.261950 & $\begin{array}{l}\text { Nesting or feeding area of Larus dominicanus: Sanionia } \\
\text { sp., limpet shells }\end{array}$ \\
\hline Winter Island & 2020 & -65.248120 & -64.264120 & Alga subformation (Prasiola crispa) \\
\hline Skua Island & 2020 & -65.252210 & -64.259860 & $\begin{array}{l}\text { Bryophyte carpet and mat subformation (Sanionia sp.) } \\
\text { and green macroalgae (Prasiola crispa) }\end{array}$ \\
\hline $\begin{array}{l}\text { un-named island (1) of the } \\
\text { Yalour Islands }\end{array}$ & 2020 & -65.236610 & -64.161740 & Bryophyte carpet and mat subformation (Sanionia sp.) \\
\hline $\begin{array}{l}\text { un-named island (1) of the } \\
\text { Yalour Islands }\end{array}$ & 2020 & -65.235320 & -64.158760 & $\begin{array}{l}\text { Bryophyte carpet and mat subformation (Sanionia sp.) } \\
\text { and penguin feathers }\end{array}$ \\
\hline $\begin{array}{l}\text { un-named island (1) of the } \\
\text { Yalour Islands }\end{array}$ & 2020 & -65.233840 & -64.161710 & Bryophyte carpet and mat subformation (Sanionia sp.) \\
\hline $\begin{array}{l}\text { un-named island (1) of the } \\
\text { Yalour Islands }\end{array}$ & 2020 & -65.235500 & -64.155760 & Bryophyte carpet and mat subformation (Sanionia sp.) \\
\hline Two Hummock Island & 2020 & -64.171240 & -61.718690 & $\begin{array}{l}\text { Bryophyte carpet and mat subformation (Sanionia sp. } \\
\text { and Warnstorfia fontinaliopsis) }\end{array}$ \\
\hline Cobalescou Island & 2020 & -64.179910 & -61.649790 & Bryophyte carpet and mat subformation (Sanionia sp.) \\
\hline Murray Island & 2020 & -64.394356 & -61.496695 & Bryophyte carpet and mat subformation (Sanionia sp.) \\
\hline Lecointe Island & 2020 & -64.23938 & -62.01304 & $\begin{array}{l}\text { Bryophyte carpet and mat subformation } \\
\text { (Warnstorfia fontinaliopsis) }\end{array}$ \\
\hline Green Point & 2020 & -64.40824 & -61.51831 & $\begin{array}{l}\text { Bryophyte carpet and mat subformation } \\
\text { (Warnstorfia fontinaliopsis) }\end{array}$ \\
\hline $\begin{array}{l}\text { un-named island near } \\
\text { Enterprise Island }\end{array}$ & 2020 & -64.540220 & -61.983385 & $\begin{array}{l}\text { Nesting or feeding area of Larus dominicanus: } \\
\text { undetermined Bryophytes }\end{array}$ \\
\hline Bryde Island & 2020 & -64.87614 & -62.92574 & Bryophyte carpet and mat subformation (Sanionia sp.) \\
\hline Spigot Point & 2020 & -64.624631 & -62.554717 & $\begin{array}{l}\text { Bryophyte carpet and mat subformation (Sanionia sp. } \\
\text { and Warnstorfia fontinaliopsis) }\end{array}$ \\
\hline $\begin{array}{l}\text { Jougla Point, Wiencke } \\
\text { Island }\end{array}$ & 2020 & -64.829300 & -63.495360 & $\begin{array}{l}\text { Bryophyte carpet and mat subformation (Sanionia sp. } \\
\text { and Warnstorfia fontinaliopsis) }\end{array}$ \\
\hline $\begin{array}{l}\text { Jougla Point, Wiencke } \\
\text { Island }\end{array}$ & 2020 & -64.82907 & -63.48868 & $\begin{array}{l}\text { Bryophyte carpet and mat subformation (Sanionia sp.), } \\
\text { with Polytrichum strictum, Prasiola crispa }\end{array}$ \\
\hline Doumer Island & 2020 & -64.833350 & -63.577590 & Bryophyte carpet and mat subformation (Sanionia sp.) \\
\hline Cape Garcia & 2020 & -65.73547 & -64.63110 & $\begin{array}{l}\text { Bryophyte carpet and mat subformation (Sanionia sp. } \\
\text { and Warnstorfia fontinaliopsis) }\end{array}$ \\
\hline Lahille Island & 2020 & -65.553605 & -64.395064 & Bryophyte carpet and mat subformation (Sanionia sp.) \\
\hline $\begin{array}{l}\text { un-named island close to } \\
\text { Enterprise Island }\end{array}$ & 2020 & -64.55345 & -61.99451 & Bryophyte carpet and mat subformation (Sanionia $\mathrm{sp.)}$ \\
\hline Duchaylard Island & 2020 & -65.72158 & -65.08225 & $\begin{array}{l}\text { Bryophyte carpet and mat subformation (Sanionia sp. } \\
\text { dominates, Warnstorfia fontinaliopsis) }\end{array}$ \\
\hline Duchaylard Island & 2020 & -65.72158 & -65.08225 & $\begin{array}{l}\text { Nesting or feeding area of Larus dominicanus: Sanionia } \\
\text { sp., limpet shells }\end{array}$ \\
\hline
\end{tabular}


P. Kovalenko, V. Trokhymets, I. Parnikoza et al.: Distribution of B. antarctica by the data of UAEs

Continuation of Table 1

\begin{tabular}{|c|c|c|c|c|}
\hline Site & Year & Latitude & Longitude & Biotope \\
\hline $\begin{array}{l}\text { Cape Evensen, Stresher } \\
\text { Peninsula, opposite the } \\
\text { Marie Island }\end{array}$ & 2020 & -66.143998 & -65.726981 & $\begin{array}{l}\text { Bryophyte carpet and mat subformation (Sanionia sp. } \\
\text { and Warnstorfia fontinaliopsis) }\end{array}$ \\
\hline Hovgaard Island & 2021 & -65.141150 & -64.095030 & Bryophyte carpet and mat subformation (Sanionia sp.) \\
\hline Hovgaard Island & 2021 & -65.140720 & -64.097980 & Bryophyte carpet and mat subformation (Sanionia sp.) \\
\hline Hovgaard Island & 2021 & -65.140220 & -64.097180 & Bryophyte carpet and mat subformation (Sanionia sp.) \\
\hline Hovgaard Island & 2021 & -65.141150 & -64.095030 & Bryophyte carpet and mat subformation (Sanionia sp.) \\
\hline Petermann Island & 2021 & -65.177167 & -64.145183 & Bryophyte carpet and mat subformation (Sanionia sp.) \\
\hline Petermann Island & 2021 & -65.172567 & -64.134700 & Bryophyte carpet and mat subformation (Sanionia sp.) \\
\hline Petermann Island & 2021 & -65.177100 & -64.143633 & Bryophyte carpet and mat subformation (Sanionia sp.) \\
\hline Petermann Island & 2021 & -65.178383 & -64.144483 & Bryophyte carpet and mat subformation (Sanionia sp.) \\
\hline Petermann Island & 2021 & -65.172333 & -64.137833 & Bryophyte carpet and mat subformation (Sanionia sp.) \\
\hline Petermann Island & 2021 & -65.177100 & -64.143633 & Bryophyte carpet and mat subformation (Sanionia sp.) \\
\hline Petermann Island & 2021 & -65.172567 & -64.134700 & Bryophyte carpet and mat subformation (Sanionia sp.) \\
\hline Petermann Island & 2021 & -65.172567 & -64.134700 & Bryophyte carpet and mat subformation (Sanionia sp.) \\
\hline Rasmussen Point & 2021 & -65.247889 & -64.084056 & Bryophyte carpet and mat subformation (Sanionia sp.) \\
\hline Rasmussen Point & 2021 & -65.247917 & -64.083611 & Bryophyte carpet and mat subformation (Sanionia sp.) \\
\hline Rasmussen Point & 2021 & -65.247778 & -64.084556 & Bryophyte carpet and mat subformation (Sanionia sp.) \\
\hline Cape Tuxen & 2021 & -65.268139 & -64.116083 & Bryophyte carpet and mat subformation (Sanionia sp.) \\
\hline Cape Tuxen & 2021 & -65.267667 & -64.116583 & Bryophyte carpet and mat subformation (Sanionia sp.) \\
\hline Skua Island & 2021 & -65.250194 & -64.273500 & Bryophyte carpet and mat subformation (Sanionia sp.) \\
\hline Skua Island & 2021 & -65.250194 & -64.273500 & Bryophyte carpet and mat subformation (Sanionia sp.) \\
\hline Skua Island & 2021 & -65.250194 & -64.273500 & Bryophyte carpet and mat subformation (Sanionia sp.) \\
\hline Galindez Island & 2021 & -65.248194 & -64.246139 & Bryophyte carpet and mat subformation (Sanionia sp.) \\
\hline Galindez Island & 2021 & -65.248194 & -64.246139 & Bryophyte carpet and mat subformation (Sanionia sp.) \\
\hline Galindez Island & 2021 & -65.248194 & -64.246139 & Bryophyte carpet and mat subformation (Sanionia sp.) \\
\hline Petermann Island & 2021 & -65.178383 & -64.144483 & Bryophyte carpet and mat subformation (Sanionia sp.) \\
\hline Winter Island & 2021 & -65.247833 & -64.267917 & Bryophyte carpet and mat subformation (Sanionia sp.) \\
\hline Winter Island & 2021 & -65.247472 & -64.258722 & Bryophyte carpet and mat subformation (Sanionia sp.) \\
\hline Winter Island & 2021 & -65.247222 & -64.255667 & Bryophyte carpet and mat subformation (Sanionia sp.) \\
\hline Winter Island & 2021 & -65.247806 & -64.265361 & Bryophyte carpet and mat subformation (Sanionia sp.) \\
\hline Rasmussen Point & 2021 & -65.247917 & -64.083611 & Bryophyte carpet and mat subformation (Sanionia sp.) \\
\hline Grotto Island & 2021 & -65.239389 & -64.253750 & Bryophyte carpet and mat subformation (Sanionia sp.) \\
\hline Corner Island & 2021 & -65.245083 & -64.232889 & Bryophyte carpet and mat subformation (Sanionia sp.) \\
\hline Corner Island & 2021 & -65.244167 & -64.227917 & Bryophyte carpet and mat subformation (Sanionia sp.) \\
\hline Uruguay Island & 2021 & -65.234167 & -64.221583 & Bryophyte carpet and mat subformation (Sanionia sp.) \\
\hline Uruguay Island & 2021 & -65.234167 & -64.221583 & Bryophyte carpet and mat subformation (Sanionia sp.) \\
\hline Pléneau Island & 2021 & -65.099722 & -64.054000 & Bryophyte carpet and mat subformation (Sanionia sp.) \\
\hline Pléneau Island & 2021 & -65.099722 & -64.054000 & Bryophyte carpet and mat subformation (Sanionia sp.) \\
\hline
\end{tabular}

ISSN 1727-7485. Український антарктичний журнал, 2021, № 2, https://doi.org/10.33275/1727-7485.2.2021.679 
End of Table 1

\begin{tabular}{|c|c|c|c|c|}
\hline Site & Year & Latitude & Longitude & Biotope \\
\hline Galindez Island & 2021 & -65.247389 & -64.253361 & Bryophyte carpet and mat subformation (Sanionia sp.) \\
\hline Galindez Island & 2021 & -65.247361 & -64.250278 & Bryophyte carpet and mat subformation (Sanionia sp.) \\
\hline Galindez Island & 2021 & -65.250833 & -64.242083 & Bryophyte carpet and mat subformation (Sanionia sp.) \\
\hline $\begin{array}{l}\text { un-named island (1) } \\
\text { of The Barchans }\end{array}$ & 2021 & -65.240556 & -64.305222 & Bryophyte carpet and mat subformation (Sanionia sp.) \\
\hline Edge Hill & 2019 & -65.238056 & -64.070000 & Bryophyte carpet and mat subformation (Sanionia sp.) \\
\hline Galindez Island & 2019 & -65.247819 & -64.243342 & Bryophyte carpet and mat subformation (Sanionia sp.) \\
\hline Galindez Island & 2019 & -65.248333 & -64.238889 & Bryophyte carpet and mat subformation (Sanionia sp.) \\
\hline Rasmussen Point & 2020 & -65.247222 & -64.084722 & Bryophyte carpet and mat subformation (Sanionia sp.) \\
\hline Fanfare Island & 2020 & -65.215556 & -64.189167 & Bryophyte carpet and mat subformation (Sanionia sp.) \\
\hline $\begin{array}{l}\text { un-named island (1) of the } \\
\text { Yalour Islands }\end{array}$ & 2020 & -65.233611 & -64.159722 & Bryophyte carpet and mat subformation (Sanionia sp.) \\
\hline King George Island & 2020 & -62.195750 & -58.961267 & Alga subformation (Prasiola crispa) \\
\hline Half Moon Island & 2020 & -62.595883 & -59.897083 & Alga subformation (Prasiola crispa) \\
\hline Deception Island & 2020 & -62.979117 & -60.577450 & Alga subformation (Prasiola crispa) \\
\hline Deception Island & 2020 & -62.979000 & -60.555917 & Alga subformation (Prasiola crispa) \\
\hline $\begin{array}{l}\text { Lécuyer Point, Wiencke } \\
\text { Island }\end{array}$ & 2020 & -64.829467 & -63.489517 & Alga subformation (Prasiola crispa) \\
\hline $\begin{array}{l}\text { un-named island (2) of the } \\
\text { Yalour Islands }\end{array}$ & 2020 & -65.242933 & -64.156367 & Alga subformation (Prasiola crispa) \\
\hline Galindez Island & 2020 & -65.246165 & -64.248986 & Bryophyte carpet and mat subformation (Sanionia sp.) \\
\hline Galindez Island & 2020 & -65.246369 & -64.247143 & Bryophyte carpet and mat subformation (Sanionia sp.) \\
\hline Galindez Island & 2020 & -65.248328 & -64.246293 & Bryophyte carpet and mat subformation (Sanionia sp.) \\
\hline Cape Tuxen & 2012 & -65.282435 & -64.100000 & Bryophyte carpet and mat subformation (Sanionia sp.) \\
\hline Cape Tuxen & 2012 & -65.16155 & -64.07443 & Bryophyte carpet and mat subformation (Sanionia sp.) \\
\hline
\end{tabular}

* Numbers in brackets near un-named islands mean that samples were collected on different un-named islands from one archipelago. 
Table 2. Information about sites for which Belgica antarctica was not recorded during XII (2007-2008), XIV (2009-2010), XVI (2011-2012), XXIV (2019-2020), and XXV (2020-2021) Ukrainian Antarctic Expeditions

\begin{tabular}{|c|c|c|c|c|}
\hline Site & Year & Latitude & Longitude & Biotope \\
\hline Galindez Island & 2007 & -65.251000 & -64.242633 & Bryophyte carpet and mat subformation (Sanionia sp.) \\
\hline Galindez Island & 2007 & -65.246367 & -64.246667 & Bryophyte carpet and mat subformation (Sanionia sp.) \\
\hline Galindez Island & 2007 & -65.248050 & -64.241700 & Bryophyte carpet and mat subformation (Sanionia sp.) \\
\hline Galindez Island & 2007 & -65.247833 & -64.252900 & Bryophyte carpet and mat subformation (Sanionia sp.) \\
\hline Petermann Island & 2007 & -65.171217 & -64.136500 & Bryophyte carpet and mat subformation (Sanionia sp.) \\
\hline Petermann Island & 2007 & -65.174233 & -64.140633 & Bryophyte carpet and mat subformation (Sanionia sp.) \\
\hline Petermann Island & 2007 & -65.178300 & -64.140650 & Bryophyte carpet and mat subformation (Sanionia sp.) \\
\hline Petermann Island & 2007 & -65.178383 & -64.144483 & Bryophyte carpet and mat subformation (Sanionia sp.) \\
\hline Petermann Island & 2007 & -65.178017 & -64.146117 & Bryophyte carpet and mat subformation (Sanionia sp.) \\
\hline Petermann Island & 2007 & -65.174350 & -64.142267 & Bryophyte carpet and mat subformation (Sanionia sp.) \\
\hline Petermann Island & 2007 & -65.177733 & -64.146750 & Bryophyte carpet and mat subformation (Sanionia sp.) \\
\hline Rasmussen Point & 2007 & -65.247750 & -64.083600 & Bryophyte carpet and mat subformation (Sanionia sp.) \\
\hline Uruguay Island & 2007 & -65.240133 & -64.229950 & Bryophyte carpet and mat subformation (Sanionia sp.) \\
\hline Berthelot Island & 2007 & -65.327583 & -64.143667 & Bryophyte carpet and mat subformation (Sanionia sp.) \\
\hline Berthelot Island & 2007 & -65.328900 & -64.162633 & Bryophyte carpet and mat subformation (Sanionia sp.) \\
\hline Booth Island & 2007 & -65.066850 & -64.023800 & Bryophyte carpet and mat subformation (Sanionia sp.) \\
\hline Skua Island & 2007 & -65.255150 & -64.273117 & Bryophyte carpet and mat subformation (Sanionia sp.) \\
\hline Skua Island & 2007 & -65.255150 & -64.274783 & Bryophyte carpet and mat subformation (Sanionia sp.) \\
\hline $\begin{array}{l}\text { un-named island of the } \\
\text { Lippmann Islands }\end{array}$ & 2008 & -65.497967 & -64.412600 & Bryophyte carpet and mat subformation (Sanionia sp.) \\
\hline King George Island & 2020 & -62.194954 & -58.966338 & Short moss turf and cushion (undetermined Bryophytes) \\
\hline King George Island & 2020 & -62.195135 & -58.966991 & $\begin{array}{l}\text { Bryophyte carpet and mat subformation (Sanionia sp.) } \\
\text { with add of Polytrichum strictum }\end{array}$ \\
\hline King George Island & 2020 & -62.193457 & -58.971036 & $\begin{array}{l}\text { Bryophyte carpet and mat subformation (Sanionia sp.) } \\
\text { with add of undetermined Bryophytes }\end{array}$ \\
\hline King George Island & 2020 & -62.189115 & -58.968600 & Bryophyte carpet and mat subformation (Sanionia sp.) \\
\hline Cape Legoupil & 2020 & -63.322024 & -57.899281 & Alga subformation (Prasiola crispa) with fur of seals \\
\hline $\begin{array}{l}\text { Mount Scott, Kyiv } \\
\text { Peninsula }\end{array}$ & 2020 & -65.153476 & -64.072531 & Bryophyte carpet and mat subformation (Sanionia sp.) \\
\hline $\begin{array}{l}\text { Coast of Kyiv Peninsula } \\
\text { near Girard Bay }\end{array}$ & 2020 & -65.129067 & -63.944600 & Bryophyte carpet and mat subformation (Sanionia sp.) \\
\hline $\begin{array}{l}\text { un-named island (1)* } \\
\text { of The Barchans }\end{array}$ & 2020 & -65.241830 & -64.300590 & $\begin{array}{l}\text { Fruticose lichen and moss cushion subformation (Usnea } \\
\text { antarctica) }\end{array}$ \\
\hline $\begin{array}{l}\text { Eastern of the Three Little } \\
\text { Pigs Islands }\end{array}$ & 2020 & -65.243405 & -64.270425 & $\begin{array}{l}\text { Short moss turf and cushion (undetermined Bryophytes), } \\
\text { limpet shells }\end{array}$ \\
\hline $\begin{array}{l}\text { Western of the Three Little } \\
\text { Pigs Islands }\end{array}$ & 2020 & -65.243770 & -64.279290 & $\begin{array}{l}\text { Fruticose lichen and moss cushion subformation } \\
\text { (Usnea antarctica) }\end{array}$ \\
\hline Skua Island & 2020 & -65.255180 & -64.274500 & $\begin{array}{l}\text { Fragment of tall moss turf subformation } \\
\text { (Polytrichum strictum) }\end{array}$ \\
\hline Skua Island & 2020 & -65.255180 & -64.274500 & Short moss turf and cushion (undetermined Bryophytes) \\
\hline $\begin{array}{l}\text { un-named island close to } \\
\text { Winter Island }\end{array}$ & 2020 & -65.254420 & -64.274930 & $\begin{array}{l}\text { Fruticose lichen and moss cushion subformation (Us- } \\
\text { nea antarctica, Xanthoria sp., and other undetermined } \\
\text { lichens) }\end{array}$ \\
\hline
\end{tabular}




\begin{tabular}{|c|c|c|c|c|}
\hline Site & Year & Latitude & Longitude & Biotope \\
\hline $\begin{array}{l}\text { un-named island close to } \\
\text { Winter Island }\end{array}$ & 2020 & -65.254420 & -64.274930 & $\begin{array}{l}\text { Fruticose lichen and moss cushion subformation }(U s- \\
\text { nea antarctica, Xanthoria sp., and other undetermined } \\
\text { lichens) }\end{array}$ \\
\hline $\begin{array}{l}\text { un-named island close to } \\
\text { Winter Island }\end{array}$ & 2020 & -65.254420 & -64.274930 & $\begin{array}{l}\text { Bryophyte carpet and mat subformation with Descham- } \\
\text { psia antarctica }\end{array}$ \\
\hline $\begin{array}{l}\text { un-named island close to } \\
\text { Winter Island }\end{array}$ & 2020 & -65.254420 & -64.274930 & Alga subformation (Prasiola crispa) \\
\hline Indicator Island & 2020 & -65.246020 & -64.264860 & $\begin{array}{l}\text { Short moss turf and cushion (undetermined Bryophy- } \\
\text { tes), limpet shells }\end{array}$ \\
\hline $\begin{array}{l}\text { un-named island (2), } \\
\text { Forge Islands }\end{array}$ & 2020 & -65.237670 & -64.273600 & $\begin{array}{l}\text { Nesting or feeding area of Larus dominicanus: Sanionia } \\
\text { sp., limpet shells, and penguin feathers }\end{array}$ \\
\hline Green Island & 2020 & -65.322700 & -64.150970 & $\begin{array}{l}\text { Bryophyte carpet and mat subformation (Warnstorfia } \\
\text { fontinaliopsis) }\end{array}$ \\
\hline Berthelot Island & 2020 & -65.336800 & -64.174600 & $\begin{array}{l}\text { Bryophyte carpet and mat subformation (Warnstorfia } \\
\text { fontinaliopsis) }\end{array}$ \\
\hline Galindez Island & 2020 & -65.245500 & -64.249520 & $\begin{array}{l}\text { Nesting or feeding area of Larus dominicanus: Sanionia } \\
\text { sp., limpet shells }\end{array}$ \\
\hline Galindez Island & 2020 & -65.245500 & -64.249520 & $\begin{array}{l}\text { Bryophyte carpet and mat subformation with Descham- } \\
\text { psia antarctica }\end{array}$ \\
\hline Galindez Island & 2020 & -65.246160 & -64.248270 & $\begin{array}{l}\text { Bryophyte carpet and mat subformation (Sanionia sp. } \\
\text { and undetermined Bryophytes) }\end{array}$ \\
\hline Galindez Island & 2020 & -65.246160 & -64.248270 & $\begin{array}{l}\text { Fragment of tall moss turf subformation (Polytrichum } \\
\text { strictum and undetermined Bryophytes) }\end{array}$ \\
\hline Galindez Island & 2020 & -65.246160 & -64.248270 & $\begin{array}{l}\text { Bryophyte carpet and mat subformation with Sanionia } \\
\text { sp. and Deschampsia antarctica }\end{array}$ \\
\hline Galindez Island & 2020 & -65.248380 & -64.245840 & $\begin{array}{l}\text { Fragment of tall moss turf subformation (Polytrichum } \\
\text { strictum) with add of Sanionia sp. }\end{array}$ \\
\hline Nob Island & 2020 & -65.205150 & -64.314320 & Bryophyte carpet and mat subformation (Sanionia sp.) \\
\hline $\begin{array}{l}\text { un-named island close to } \\
\text { Nob Island }\end{array}$ & 2020 & -65.205900 & -64.311950 & $\begin{array}{l}\text { Bryophyte carpet and mat subformation (Sanionia sp.) } \\
\text { and limpet shells }\end{array}$ \\
\hline Galindez Island & 2020 & -65.248584 & -64.238834 & $\begin{array}{l}\text { Nesting or feeding area of Larus dominicanus: Descham- } \\
\text { psia antarctica, undetermined Bryophytes, and limpet } \\
\text { shells }\end{array}$ \\
\hline Galindez Island & 2020 & -65.245720 & -64.256820 & Bryophyte carpet and mat subformation (Sanionia sp.) \\
\hline Galindez Island & 2020 & -65.245720 & -64.256820 & $\begin{array}{l}\text { Bryophyte carpet and mat subformation (Sanionia sp. } \\
\text { and undetermined Bryophytes) }\end{array}$ \\
\hline Galindez Island & 2020 & -65.245720 & -64.256820 & $\begin{array}{l}\text { Bryophyte carpet and mat subformation with Descham- } \\
\text { psia antarctica }\end{array}$ \\
\hline Galindez Island & 2020 & -65.245720 & -64.256820 & $\begin{array}{l}\text { Bryophyte carpet and mat subformation with Descham- } \\
\text { psia antarctica }\end{array}$ \\
\hline Galindez Island & 2020 & -65.245930 & -64.257560 & $\begin{array}{l}\text { Bryophyte carpet and mat subformation (Sanionia sp. } \\
\text { and undetermined Bryophytes) }\end{array}$ \\
\hline Hovgaard Island & 2020 & -65.119340 & -64.073410 & $\begin{array}{l}\text { Bryophyte carpet and mat subformation (Warnstorfia } \\
\text { fontinaliopsis) }\end{array}$ \\
\hline Hovgaard Island & 2020 & -65.119340 & -64.073410 & $\begin{array}{l}\text { Fruticose lichen and moss cushion subformation (Usn- } \\
\text { ea antarctica and Umbilicaria sp.) }\end{array}$ \\
\hline Petermann Island & 2020 & -65.165430 & -64.142670 & $\begin{array}{l}\text { Bryophyte carpet and mat subformation (Sanionia sp. } \\
\text { and undetermined Bryophytes), limpet shells }\end{array}$ \\
\hline
\end{tabular}


P. Kovalenko, V. Trokhymets, I. Parnikoza et al.: Distribution of B. antarctica by the data of UAEs

End of Table 2

\begin{tabular}{|c|c|c|c|c|}
\hline Site & Year & Latitude & Longitude & Biotope \\
\hline Torgersen Island & 2020 & -64.771580 & -64.074220 & $\begin{array}{l}\text { Bryophyte carpet and mat subformation (Sanionia sp. } \\
\text { and undetermined Bryophytes) and green macroalgae } \\
\text { (Prasiola crispa) }\end{array}$ \\
\hline Cape Tuxen & 2020 & -65.267660 & -64.115930 & Short moss turf and cushion (undetermined Bryophytes) \\
\hline Rasmussen Island & 2020 & -65.257190 & -64.077070 & $\begin{array}{l}\text { Bryophyte carpet and mat subformation (Sanionia sp. } \\
\text { and undetermined Bryophytes) }\end{array}$ \\
\hline Rasmussen Point & 2020 & -65.247810 & -64.084690 & $\begin{array}{l}\text { Bryophyte carpet and mat subformation (Warnstorfia } \\
\text { fontinaliopsis), green macroalgae (Prasiola crispa) }\end{array}$ \\
\hline Winter Island & 2020 & -65.248320 & -64.266020 & Bryophyte carpet and mat subformation (Sanionia sp.) \\
\hline Deception Island & 2020 & -62.982130 & -60.518980 & Bryophyte carpet and mat subformation (Sanionia sp.) \\
\hline Two Hummock Island & 2020 & -64.171240 & -61.718690 & $\begin{array}{l}\text { Bryophyte carpet and mat subformation (Warnstorfia } \\
\text { fontinaliopsis and Sanionia sp.) and Usnea antarctica }\end{array}$ \\
\hline Eckener Point & 2020 & -64.429295 & -61.599018 & Bryophyte carpet and mat subformation (Sanionia sp.) \\
\hline Cuverville Island & 2020 & -64.684360 & -62.622660 & Fragment of tall moss turf subformation (Polytrichum strictum) \\
\hline Cuverville Island & 2020 & -64.68436 & -62.62266 & $\begin{array}{l}\text { Bryophyte carpet and mat subformation (Warnstorfia } \\
\text { fontinaliopsis) }\end{array}$ \\
\hline Wienckie Island & 2020 & -64.825460 & -63.481160 & $\begin{array}{l}\text { Bryophyte carpet and mat subformation (Sanionia sp. } \\
\text { and undetermined Bryophytes), limpet shells }\end{array}$ \\
\hline False Cape Renard & 2020 & -65.02776 & -63.81460 & Bryophyte carpet and mat subformation (Sanionia sp.) \\
\hline Lagoon Island & 2020 & -67.59465 & -68.23127 & $\begin{array}{l}\text { Nesting or feeding area of Larus dominicanus: Polytrichum } \\
\text { strictum, Sanionia sp., Deschampsia antarctica, limpet } \\
\text { shells, and penguin feathers }\end{array}$ \\
\hline Hook Island & 2020 & -65.638110 & -65.172240 & $\begin{array}{l}\text { Fragment of tall moss turf subformation (Polytrichum stric- } \\
\text { tum and undetermined Bryophytes) with add of Sanionia sp. }\end{array}$ \\
\hline un-named island, Pitt Islands & 2020 & -65.43937 & -65.35348 & Bryophyte carpet and mat subformation (Sanionia sp.) \\
\hline Lagotellerie Island & 2020 & -67.88486 & -67.38765 & Alga subformation (Prasiola crispa) with fur of seals \\
\hline $\begin{array}{l}\text { Camp Point on Fallières } \\
\text { Coast }\end{array}$ & 2020 & -67.93874 & -67.14997 & $\begin{array}{l}\text { Bryophyte carpet and mat subformation with Descham- } \\
\text { psia antarctica }\end{array}$ \\
\hline Horseshoe Island & 2020 & -67.80884 & -67.29895 & Short moss turf and cushion (undetermined Bryophytes) \\
\hline Pourquoi Pas Island & 2020 & -67.57904 & -67.25851 & Bryophyte carpet and mat subformation (Sanionia sp.) \\
\hline Blaiklock Island & 2020 & -67.55621 & -67.20963 & $\begin{array}{l}\text { Bryophyte carpet and mat subformation with Sanionia } \\
\text { sp. and Deschampsia antarctica }\end{array}$ \\
\hline Léonie Island & 2020 & -67.59375 & -68.33702 & $\begin{array}{l}\text { Bryophyte carpet and mat subformation with Sanionia } \\
\text { sp., Warnstorfia fontinaliopsis, Deschampsia antarctica }\end{array}$ \\
\hline Arrowsmith Peninsula & 2020 & -67.504220 & -67.231650 & Bryophyte carpet and mat subformation (Sanionia sp.) \\
\hline Winter Island & 2021 & -65.250111 & -64.256250 & Bryophyte carpet and mat subformation (Sanionia sp.) \\
\hline Grotto Island & 2021 & -65.239389 & -64.253750 & Bryophyte carpet and mat subformation (Sanionia sp.) \\
\hline Galindez Island & 2021 & -65.247444 & -64.241861 & Bryophyte carpet and mat subformation (Sanionia sp.) \\
\hline Galindez Island & 2021 & -65.246139 & -64.248917 & Bryophyte carpet and mat subformation (Sanionia sp.) \\
\hline $\begin{array}{l}\text { Central of the Three Little } \\
\text { Pigs Islands }\end{array}$ & 2021 & -65.242083 & -64.274917 & Bryophyte carpet and mat subformation (Sanionia sp.) \\
\hline $\begin{array}{l}\text { Eastern of the Three Little } \\
\text { Pigs Islands }\end{array}$ & 2021 & -65.242139 & -64.271194 & Bryophyte carpet and mat subformation (Sanionia sp.) \\
\hline Indicator Island & 2021 & -65.245861 & -64.265111 & Bryophyte carpet and mat subformation (Sanionia sp.) \\
\hline
\end{tabular}

* Numbers in brackets near un-named islands mean that samples were collected on different un-named islands from one archipelago.

ISSN 1727-7485. Український антарктичний журнал, 2021, № 2, https://doi.org/10.33275/1727-7485.2.2021.679 